\title{
3. Forschungsfragen der Untersuchung
}

Ziel der Arbeit ist es, die Rolle von Auszeichnungen zur Anerkennung von Freiwilligenarbeit in Deutschschweizer Freiwilligenorganisationen zu erforschen. Dabei stellt sich zunächst die Frage, ob Auszeichnungen in Deutschschweizer Freiwilligenorganisationen zur Anerkennung von Freiwilligenarbeit eingesetzt werden und wie diese hinsichtlich der vier Parameter (Formen und Bestandteile, Periodizität und Empfänger, Sichtbarkeit und Öffentlichkeitsgrad sowie Vorgehen und Selektion) gestaltet werden. Zwei weitere Forschungsziele bestehen darin, die Wichtigkeit der Gestaltungsparameter von Auszeichnungen sowie die Wirkung von Auszeichnungen aus Sicht der Freiwilligenorganisationen zu untersuchen. Um diese Forschungsziele zu erreichen, wurden auf Basis der bisherigen Ausführungen folgende Forschungsfragen abgeleitet:

Werden Auszeichnungen zur Anerkennung von Freiwilligenarbeit in Deutschschweizer Freiwilligenorganisationen eingesetzt?

Wie schätzen die Organisationen die Wirkungen von Auszeichnungen auf die Ausgezeichneten bzw. die Nicht-Ausgezeichneten ein?

Wie werden Auszeichnungen hinsichtlich der vier Parameter Formen und Bestandteile, Periodizität und Empfänger, Sichtbarkeit und Öffentlichkeitsgrad sowie Vorgehen und Selektion gestaltet?

| Wie beurteilen die Organisationen die Wichtigkeit der vier Gestaltungsparameter von Auszeichnungen? 